\title{
Immature oocyte incidence: Contributing factors and effects on mature sibling oocytes in intracytoplasmic sperm injection cycles
}

\author{
Daniela Paes de Almeida Ferreira Braga ${ }^{1,2}$, Bianca Ferrarini Zanetti ${ }^{1,2}$, Amanda Souza Setti ${ }^{1,2}$, Assumpto Iaconelli \\ $\mathrm{Jr}^{1,2}$, Edson Borges Jr. ${ }^{1,2}$ \\ ${ }^{1}$ Fertility Medical Group, São Paulo, SP - Brazil \\ ${ }^{2}$ Instituto Sapientiae - Centro de Estudos e Pesquisa em Reprodução Humana Assistida, São Paulo, SP - Brazil
}

\begin{abstract}
Objective: The aim of this study was to investigate which factors contribute to the incidence of immature oocytes (germinal vesicle -GV- and metaphase I -MI-) and how they impact the intracytoplasmic sperm injection (ICSI) outcomes of sibling mature oocytes.

Methods: Data from 3,920 cycles performed from June/2010 to August/2016 in a private university-affiliated IVF center were evaluated for the influence of controlled ovarian stimulation protocol (COS) on immature oocytes incidence and its effects on ICSI outcomes.

Results: MI $(p=0.004)$ and GV $(p=0.029)$ number were negatively correlated with gonadotropin dose. Patients stimulated by rFSH had increased GV/oocyte rate in both GnRH agonists $(p<0.001)$ and antagonist $(p=0.042)$ protocols, in comparison to rFSH associated with rLH protocol. $\mathrm{MI}$ and GV/oocyte rates were negatively correlated to fertilization $(p<0.001)$, high-quality embryo on da $p<0.001$; GV/oocyte $p=0.033$ ) and pregnancy (MI/oocyte $p=0.002$; GV/oocyte $p=0.013$ ) rates. Cycles above a $10.5 \% \mathrm{MI} / \mathrm{oo-}$ cyte cut-off were correlated to higher response to ovarian stimulation, poor embryo development and almost two times lower pregnancy rate. Immature oocyte incidence is affected by COS and impacts on ICSI outcomes.

Conclusion: Our evidence suggests that oocytes derived from a cohort with high incidence of maturation fail may have detrimental clinical outcomes.
\end{abstract}

Keywords: germinal vesicle, metaphase I, immature oocyte, ICSI, pregnancy

\section{INTRODUCTION}

By the time of birth, the human ovary contains a pool of quiescent primordial follicles, each consisting of a small inactive oocyte, arrested in the germinal vesicle (GV) stage, and a single layer of granulosa cells; the fate of each follicle is controlled by endocrine as well as paracrine factors (Findlay et al., 2015; Jaffe \& Egbert, 2017). Of the millions of the primordial oocytes present at birth, only approximately 400 mature during a woman's lifetime (Monniaux et al., 2014). Oocyte maturation is defined as the restart and completion of the first meiotic division from prophase I, through metaphase I (MI stage), to metaphase II (MII stage), with accompanying cytoplasmic maturation, which includes the storage of cytoplasmic enzymes, mRNAs, organelles, and metabolic substrates that are crucial for fertilization and early embryonic development (Coticchio et al., 2015; Downs, 2015).

In contrast to the in vivo process, where oocyte maturation occurs as the result of the natural selection, in assisted reproduction cycles, supra-physiologic gonadotropin doses induce multiple follicular growth and maturation, in order to ensure the maximum number of obtained embryos, and thereafter the highest probability of a successful pregnancy (Bosch \& Ezcurra, 2011; Fatemi et al., 2012). Even though controlled ovarian stimulation (COS) has been developed and refined in attempt to obtain optimal oocytes number from each cycle, different COS protocols may result in follicular asynchrony and variations in oocyte number, quality, viability, and competence (Jungheim et al., 2015).

Usually, 10 to $30 \%$ of oocytes are still immature at retrieval (GV or MI stages) (Borges et al., 2016; 2017). Some of the immature oocytes may extrude the first polar body and progress to maturity during in vitro culture, and might be considered for sperm injection, while others, especially those at the GV stage, will not mature during the observation time (Shu et al., 2007; Braga et al., 2010).

The asynchronous ovarian response, with numerous immature oocytes at ovum pick-up, can be an indicative that overall ovarian follicles were less responsive to ovarian stimulation, and that oocytes considered mature in the same cohort may not be fully competent for fertilization and embryo formation (Devreker et al., 1999; Halvaei et al., 2012). It has been suggested that maturation fail may lead to anomalies such as multinucleation and aneuploidy, which directly impacts the developmental competence of embryos (Nogueira et al., 2000; Emery et al., 2005).

As there are no macroscopic markers and no single observable factor indicating cytoplasmic maturation completion, there are scarce data about the probable impact of higher immature oocytes incidence in the developmental competence of mature oocytes from same cohort. Therefore, the goal for the present study was to investigate which factors contribute to the incidence of immature oocytes and how immature oocytes impacts on the outcomes of mature oocytes from the same cohort.

\section{MATERIALS AND METHODS}

\section{Experimental design}

This historical cohort study included data from 26,040 oocytes obtained from 3,920 cycles performed from June/2010 to August/2016, in a private university-affiliated IVF center. The inclusion criterion was couples undergoing first COS and ICSI cycle with fresh embryo transfer at day five. The exclusion criteria were as follows: ICSI cycle with vitrified/thawed or donated oocytes, surgical sperm retrieval, vitrified/thawed embryo transfer, or pre-implantation genetic testing.

The influence of COS protocol, pituitary suppression protocol, estradiol level on the day of hCG trigger, and interval between hCG trigger and oocyte retrieval on the numbers and rates of immature oocytes were evaluated. 
The effects of immature oocytes rates on fertilization rate, high-quality embryos rates on cleavage-stage (days two and three), blastocyst rate, implantation rate, pregnancy rate and miscarriage rate were also analyzed.

All patients signed a written informed consent form and the study was approved by the local institutional review board.

\section{Controlled ovarian stimulation}

COS was achieved by the administration of daily doses of recombinant FSH (Gonal- $F^{\circledR}$, Merck KGaA, Geneva, Switzerland); or recombinant FSH associated with recombinant LH (Gonal- $F^{\circledR}$ and Pergoveris ${ }^{\circledR}$, Merck KGaA) beginning on day three of the cycle. Pituitary suppression was performed by $\mathrm{GnRH}$ antagonist (Cetrotide ${ }^{\circledR}$; Merck KGaA) beginning when at least one follicle $\geq 14 \mathrm{~mm}$ was visualized, or GnRH agonist (Lupron Kit ${ }^{\mathrm{TM}}$, Abbott Laboratoires, Paris, France), from day 21 of the anterior menstrual cycle.

Follicular growth was monitored by transvaginal ultrasound examination. When $\geq 3$ follicles attained a mean diameter of $\geq 17 \mathrm{~mm}$ and adequate serum estradiol levels were observed, recombinant hCG (Ovidrel ${ }^{\circledR}$, Serono) was administered to trigger final follicular maturation. Ovum pick-up through transvaginal ultrasound was scheduled for 34-36 hours after hCG administration. The interval time between hCG administration and beginning of follicular aspiration was annotated. Oocyte yield was defined as the number of retrieved oocytes by the number of aspirated follicles.

\section{Oocyte preparation}

Retrieved oocytes were maintained in culture media (Global for fertilization, LifeGlobal, Guilford, USA) supplemented with $10 \%$ protein (LGPS, LifeGlobal) and covered with paraffin oil (Paraffin oil P.G., LifeGlobal) for $4 \mathrm{~h}$ before cumulus cell removal. The cumulus cells were removed after exposure to a HEPES-buffered medium containing hyaluronidase ( $80 \mathrm{IU} / \mathrm{ml}$, LifeGlobal). The remaining cells were mechanically removed by a hand-drawn Pasteur pipette (Humagen Fertility Diagnostics, Charlottesville, USA).

Oocyte morphology and maturation stage were assessed using an inverted Nikon Diaphot microscope with a Hoffmann modulation contrast system under 400x magnification (Eclipse TE 300 microscope, Nikon, Tokyo, Japan), just before sperm injection (5-7 hours after retrieval). Oocytes that had released the first polar body were considered mature (MII stage) and were submitted to ICSI.

Immature oocyte rates were defined by cycle as the number of MI oocytes by the total number of retrieved oocytes (MI/oocyte) and as the as number of GV oocytes by the total number of retrieved oocytes (GV/oocyte).

\section{Intracytoplasmic sperm injection}

Intracytoplasmic sperm injection was performed according to Palermo et al. (1992). Fertilization was confirmed approximately $16 \mathrm{~h}$ after ICSI. Embryos were maintained in a $50-\mu \mathrm{L}$ drop of culture medium (Global ${ }^{\circledR}$, LifeGlobal) with $10 \%$ protein supplement and covered with paraffin oil in a humidified atmosphere under $6 \% \mathrm{CO}_{2}$ at $37^{\circ} \mathrm{C}$ for five days.

\section{Embryo quality and embryo transfer}

Cleavage-stage morphology on days two and three were evaluated according to Istanbul consensus (Alpha Scientists in Reproductive Medicine \& ESHRE Special Interest Group of Embryology, 2011). High-quality cleavage-stage embryos were defined as those with all of the following characteristics: three to five cells on day two or eight to ten cells on day three, $<10 \%$ fragmentation, symmetric blastomeres, absence of multinucleation, colorless cytoplasm with moderate granulation and no inclusions, the absence of perivitelline space granularity and the absence of zona pellucida dimorphisms. Embryos lacking any of these characteristics were considered to be of low quality. The blastocyst rate was defined as the number of embryos that reached blastocyst stage at day five by the number of 2PN embryos.

Embryo transfer was performed on the fifth day of development using a soft catheter with transabdominal ultrasound guidance. Considering their morphology, one to two embryos were transferred per patient.

\section{Clinical follow-up}

A pregnancy test was performed 10 days after embryo transfer. All women with a positive test received a transvaginal ultrasound scan after two weeks. Clinical pregnancy was diagnosed when fetal heartbeat was detected. Implantation rates were calculated per patient as the number of gestational sacs by the number of transferred embryos. Pregnancy rates were calculated per embryo transfer. Miscarriage was defined as a pregnancy loss before 20 weeks.

\section{Data analysis}

The sample size calculation using G*Power 3.1.7 (Franz Faul, Universität Kiel, Germany) suggested that 2,848 cycles would be enough to demonstrate a $10 \%$ effect with $95 \%$ power and $5 \%$ significance level. The analysis was performed using SPSS Statistics 21 (IBM, New York, New York, USA).

The correlation between GV/oocyte and MI/oocyte rates was evaluated by Pearson ${ }^{\circledR}$.

To assess the contributing factors to immature oocyte incidence, total gonadotropin dose administered, estradiol level on the day of hCG administration, and interval between hCG trigger and oocyte retrieval were used as predictors in linear regression models. The models were adjusted for maternal age and maternal body mass index (BMI). The effects of COS protocol (rFSH vs. rFSH plus $\mathrm{rLH}$ ) and pituitary suppression (GnRH agonist vs. antagonist) were evaluated by Generalized Linear Model followed by Bonferroni post hoc, adjusted for maternal age, maternal BMI and total gonadotropin dose.

To assess the correlation of immature oocyte rates and ICSI outcomes of mature oocytes from the same cohort, laboratorial outcomes (fertilization rate, high-quality embryos rates on day two and three, and blastocyst rate) were analyzed by linear regression models adjusted for maternal age, maternal BMI, total FSH dose, estradiol level on the day of hCG administration and number of retrieved oocytes. The correlation of immature oocyte rates and clinical outcomes (implantation, pregnancy, and miscarriage rates) were assessed by linear and binary logistic regression models adjusted for maternal age, maternal BMI, total FSH dose, estradiol level on the day of hCG administration, number of retrieved oocytes, number of transferred embryos and endometrial thickness.

Discriminant Analysis for pregnancy outcome prediction (positive vs. negative) was performed using as independent variables: MI/oocyte and GV/oocyte rates, maternal age, maternal BMI, total FSH dose, estradiol level on the day of hCG administration, number of retrieved oocytes, number of transferred embryos, and endometrial thickness. The data was grouped according with established cut-off for MI/oocyte rate, and the analysis were performed by Generalized Linear Model followed by Bonferroni post hoc, adjusted for the same confounders variables described above. 


\section{RESULTS}

The descriptions of patients' characteristics and ICSI outcomes are shown in Table 1. Although statistically significant, the correlation coefficient between GV/oocyte and MI/oocyte rates was low (Pearson's $r=-0.079 p<0.001$ ), therefore each rate was evaluated separately.

The total dose of gonadotropin administered negatively affected the number of MI $(p=0.004)$, and GV oocytes $(p=0.029)$. Higher estradiol concentration at hCG day was correlated to higher number of MI $(p<0.001)$, and GV $(p<0.001)$ oocytes. The interval between hCG trigger and oocyte retrieval was not correlated to the number or rate of immature oocytes (Table 2).

In patients undergoing $\mathrm{GnRH}$ agonist protocols, GV/ oocyte rate was increased $(p<0.001)$ in patients receiving rFSH in comparison to rFSH plus rLH. Likewise, in GnRH antagonist regimens, the rFSH plus rLH stimulus was correlated to lower $\mathrm{GV} /$ oocyte rate $(p=0.042)$ in comparison to rFSH (Table 3).

The incidence of MI and GV oocytes were negatively correlated with fertilization rate $(p<0.001)$, high-quality embryos rates at days two and three $(p<0.001)$, as well as blastocyst rate $(p<0.001)$. The negative effect was also noted in the implantation (MI/oocyte $p<0.001 ; \mathrm{GV} /$ oocyte $p=0.033$ ) and pregnancy (MI/oocyte $p=0.002$; GV/oocyte $p=0.013$ ) rates (Table 4$)$.

Discriminant analyses were conducted to examine which factors would predict the pregnancy success in ICSI cycles. Mother age, number of transferred embryos, and MI/oocyte rate were the variables with higher correlation within function. The discriminant function could correctly classified $66.8 \%$ of original cases, with a better prediction for negative pregnancy, of $94.1 \%$. In this model, the MI/ oocyte rate of cycles that resulted in clinical pregnancy was of $9.56 \pm 11.25 \%$ and that of negative pregnancy of $11.49 \pm 16.00 \%(p=0.002)$. A cut-off point has been established halfway MI/oocyte rates averages, at $10.5 \%$.

Cycles with MI/oocyte above cut-off were correlated to higher response to ovarian stimulation, with higher number of follicles and oocytes $(p<0.001)$, lower fertilization rate $(p<0.001)$, lower number of obtained embryos $(p<0.001)$ and lower rate of high-quality embryos at day two $(p<0.001)$. Implantation rate was reduced $(p=0.038)$ and pregnancy rate was almost two times lower $(p<0.001)$ (Table 5).

\section{DISCUSSION}

During COS, supra-physiological environment induce multiple follicular growth and maturation of oocytes that, which under natural conditions, would regress. Oocytes retrieved are not only at different stages of nuclear maturity, but probably also cytoplasmic maturity. Our evidence suggests that mature oocytes derived from a controlled stimulated cohort with high incidence of maturation fail may have inefficient biological machinery and detrimental clinical outcomes.

The gonadotropin dose is crucial for appropriate regulation of paracrine factors that induce proliferation and differentiation of pre-antral follicles and promote oocyte developmental competence (Thomas et al., 2005; Santi et al., 2017). The duration of FSH elevation above a critical threshold level, rather than the height of the elevation of FSH for single dominant follicle selection, is a key point for the recruitment of follicles from the resting pool during COS (Messinis \& Templeton, 1990; van der Meer et al., 1994; van Santbrink et al., 1995). In fact, here we showed that ovarian hyper stimulation with higher gonadotropin doses was associated with a more homogeneous cohort development, with lower number of MI and GV immature oocytes.

\begin{tabular}{|c|c|}
\hline & Mean \pm SD \\
\hline \multicolumn{2}{|l|}{ Patient's demographics } \\
\hline Maternal age (years) & $35.99 \pm 4.68$ \\
\hline Maternal BMI $\left(\mathrm{kg} / \mathrm{m}^{2}\right)$ & $24.38 \pm 3.87$ \\
\hline Paternal age (years) & $38.50 \pm 6.47$ \\
\hline $\begin{array}{l}\text { Total gonadotropin } \\
\text { administered (IU) }\end{array}$ & $2470.26 \pm 911.26$ \\
\hline $\begin{array}{l}\text { Estradiol level at hCG trigger } \\
\text { day }(\mathrm{pg} / \mathrm{mL})\end{array}$ & $1935.24 \pm 1802.39$ \\
\hline $\begin{array}{l}\text { Interval between hCG and } \\
\text { oocyte retrieval (hours) }\end{array}$ & $35.34 \pm 0.42$ \\
\hline \multicolumn{2}{|l|}{ cos outcomes } \\
\hline Follicles (n) & $15.40 \pm 11.77$ \\
\hline Retrieved oocytes (n) & $10.51 \pm 8.41$ \\
\hline Oocyte yield (\%) & $68.87 \pm 20.09$ \\
\hline MII Oocytes (n) & $8.03 \pm 6.61$ \\
\hline MI Oocytes (n) & $1.12 \pm 1.56$ \\
\hline GV Oocytes (n) & $1.34 \pm 2.20$ \\
\hline MII/oocytes (\%) & $77.69 \pm 20.99$ \\
\hline MI/oocytes (\%) & $10.78 \pm 15.35$ \\
\hline GV/oocytes (\%) & $11.28 \pm 15.56$ \\
\hline \multicolumn{2}{|l|}{ Laboratorial outcomes } \\
\hline Fertilization rate $(\%)$ & $82.16 \pm 21.46$ \\
\hline Embryos obtained $(n)$ & $6.64 \pm 5.08$ \\
\hline $\begin{array}{l}\text { High-quality embryos rate at } \\
\text { day two }(\%)\end{array}$ & $25.74 \pm 24.60$ \\
\hline $\begin{array}{l}\text { High-quality embryos rate at } \\
\text { day three }(\%)\end{array}$ & $48.59 \pm 28.40$ \\
\hline Blastocyst rate (\%) & $47.60 \pm 32.48$ \\
\hline Transferred embryos (n) & $1.76 \pm 1.09$ \\
\hline Endometrial thickness $(\mathrm{mm})$ & $10.68 \pm 2.28$ \\
\hline \multicolumn{2}{|l|}{ Clinical outcomes } \\
\hline Implantation rate $(\%)$ & $31.83 \pm 41.36$ \\
\hline Clinical pregnancy rate $(\%)$ & 33.7 \\
\hline Miscarriage rate $(\%)$ & 14.0 \\
\hline
\end{tabular}

FSH: follicle stimulating hormone;hCG: human chorionic gonadotropin;IU: international units

In our study it was possible to correlate the COS protocol with the incidence of immature oocytes, since COS with exclusively $\mathrm{rFSH}$ was associated with higher GV/oocyte rate in comparison to rFSH associated with $r \mathrm{LH}$, in both $\mathrm{GnRH}$ agonist and antagonist pituitary suppression protocols. Pior to midcycle LH surge, which is responsible for final oocyte maturation and ovulation, the growing oocyte acquires the ability to undergo oocyte maturation while being exposed to pulsatile LH basal levels (Mehlmann, 2005; Conti et al., 2012; Celik et al., 2015; Coticchio et al., 2015). Thus, LH association to FSH during follicular development may promote a more physiologically environment, and can be a manner to improve oocyte maturation. Some authors observed that the presence of LH activity may have a positive effect on oocyte maturation (Selman 
Table 2. Contributing factors to immature oocyte incidence $(n=3,920)$

\begin{tabular}{|c|c|c|c|c|c|c|}
\hline & \multicolumn{3}{|c|}{ MI } & \multicolumn{3}{|c|}{ GV } \\
\hline & $\mathbf{R}^{2}$ & $\boldsymbol{\beta}$ & $\boldsymbol{P}$ & $\mathbf{R}^{2}$ & $\boldsymbol{\beta}$ & $\boldsymbol{p}$ \\
\hline Total gonadotropin dose & 0.042 & -0.046 & 0.004 & 0.050 & -0.035 & 0.029 \\
\hline Estradiol level at hCG trigger day & 0.146 & 0.324 & $<0.001$ & 0.155 & 0.342 & $<0.001$ \\
\hline \multirow[t]{3}{*}{ Interval between hCG and oocyte retrieval } & 0.042 & -0.015 & 0.368 & 0.050 & -0.014 & 0.385 \\
\hline & \multicolumn{3}{|c|}{ MI/oocyte } & \multicolumn{3}{|c|}{ GV/oocyte } \\
\hline & $\mathbf{R}^{2}$ & $\boldsymbol{\beta}$ & $\boldsymbol{P}$ & $\mathbf{R}^{\mathbf{2}}$ & $\boldsymbol{\beta}$ & $\boldsymbol{p}$ \\
\hline Total gonadotropin dose & 0.001 & -0.009 & 0.567 & 0.002 & 0.009 & 0.592 \\
\hline Estradiol level at hCG trigger day & 0.001 & 0.015 & 0.491 & 0.003 & 0.034 & 0.107 \\
\hline Interval between hCG and oocyte retrieval & 0.002 & -0.025 & 0.135 & 0.003 & -0.015 & 0.356 \\
\hline
\end{tabular}

hCG: human chorionic gonadotropin; $\mathrm{R}^{2}$ : effect; $\beta$ : standardized regression coefficient.

* Data was adjusted for maternal age, maternal BMI and total FSH dose.

Table 3. Effect of the pituitary suppression and COS protocol on incidence of immature oocytes

\begin{tabular}{|l|c|c|c|c|c|c|}
\hline & \multicolumn{3}{|c|}{ GnRH agonist } & \multicolumn{3}{c|}{ GnRH antagonista } \\
\hline & rFSH (n=658) & $\begin{array}{c}\text { rFSH plus rLH } \\
\text { (n=712) }\end{array}$ & $\boldsymbol{P}$ & $\begin{array}{c}\text { rFSH } \\
(\mathbf{n = 1 , 5 7 0 )}\end{array}$ & $\begin{array}{c}\text { rFSH plus rLH } \\
\text { (n=980) }\end{array}$ & $\boldsymbol{p}$ \\
\hline MI & $1.45 \pm 0.10$ & $0.38 \pm 0.68$ & 0.119 & $1.13 \pm 0.03$ & $1.12 \pm 0.05$ & 0.928 \\
\hline GV & $1.46 \pm 0.14$ & $0.40 \pm 0.93$ & 0.263 & $1.33 \pm 0.05$ & $1.36 \pm 0.08$ & 0.731 \\
\hline MI/oocyte & $13.40 \pm 0.91$ & $6.32 \pm 6.19$ & 0.147 & $10.75 \pm 0.36$ & $11.33 \pm 0.59$ & 0.405 \\
\hline GV/oocyte & $11.52 \pm 1.12$ & $1.86 \pm 2.10$ & $<\mathbf{0 . 0 0 1}$ & $11.01 \pm 0.36$ & $5.93 \pm 5.40$ & $\mathbf{0 . 0 4 2}$ \\
\hline
\end{tabular}

GnRH: gonadotropin-releasing hormone; FSH: follicle stimulating hormone; LH: luteinizing hormone.

* Data was adjusted for maternal age, maternal BMI, total FSH dose

\begin{tabular}{|c|c|c|c|c|c|c|}
\hline & \multicolumn{3}{|c|}{ MI/oocyte } & \multicolumn{3}{|c|}{ GV/oocyte } \\
\hline & $\mathbf{R}^{2}$ & $\boldsymbol{\beta}$ & $\boldsymbol{p}$ & $\mathbf{R}^{2}$ & $\boldsymbol{\beta}$ & $\boldsymbol{p}$ \\
\hline \multicolumn{7}{|l|}{ Laboratorial outcomes* } \\
\hline Fertilization rate & 0.035 & -0.096 & $<0.001$ & 0.029 & -0.059 & $<0.001$ \\
\hline High-quality embryos rate at day two & 0.014 & -0.102 & $<0.001$ & 0.008 & -0.066 & $<0.001$ \\
\hline High-quality embryos rate at day three & 0.020 & -0.090 & $<0.001$ & 0.020 & -0.087 & $<0.001$ \\
\hline Blastocyst rate & 0.073 & -0.066 & $<0.001$ & 0.071 & -0.053 & $<0.001$ \\
\hline \multicolumn{7}{|l|}{ Clinical outcomes** } \\
\hline \multirow[t]{2}{*}{ Implantation rate } & 0.059 & -0.074 & $<0.001$ & 0.056 & -0.042 & 0.033 \\
\hline & B & OR & $P$ & B & OR & $P$ \\
\hline Pregnancy rate & -0.011 & 0.989 & 0.002 & -0.009 & 0.992 & 0.013 \\
\hline Miscarriage rate & 0.010 & 1.011 & 0.220 & 0.006 & 0.944 & 0.418 \\
\hline
\end{tabular}

R2: effect; $\beta$ : standardized regression coefficient;

$\mathrm{B}$ : binary regression coefficient, OR: odds ratio for regression coefficient.

* Data was adjusted for maternal age, maternal BMI, total FSH dose, estradiol level at hCG day and number of retrieved oocytes.

** Data was adjusted for maternal age, maternal BMI, total FSH dose, estradiol level at hCG day, number of retrieved oocytes, number of transferred embryos and endometrial thickness.

et al., 2002; 2010; Pacchiarotti et al., 2007; Santi et al., 2017) and LH supplementation had been correlated to higher number of mature oocytes in COS protocols for anovulatory patients (Burgués \& Spanish Collaborative Group on Female Hypogonadotrophic, 2001; Raju et al., 2013) or patients with poor ovarian response (Papaleo et al., 2014).
Higher estradiol concentration at hCG day was highly associated with the number of immature oocytes, in accordance to previous reports (Dor et al., 1992; Suchanek et al., 1994). Although the time interval between hCG trigger and oocyte retrieval is a well-established fundamental parameter to the retrieval of mature oocytes (Raziel et al., 
Table 5. Descriptive analysis of patients' demographics and ICSI outcomes by MI/oocyte cutoff

\begin{tabular}{|c|c|c|c|}
\hline & $\begin{array}{c}\text { MI/oocyte } \\
\leq 10.5 \% \\
(n=2,399)\end{array}$ & $\begin{array}{c}\text { MI/oocyte } \\
>10.5 \% \\
(n=1,521)\end{array}$ & $\boldsymbol{p}$ \\
\hline \multicolumn{4}{|l|}{ Patient's demographics } \\
\hline Maternal age (years) & $36.10 \pm 4.82$ & $35.77 \pm 4.41$ & 0.034 \\
\hline Maternal BMI $\left(\mathrm{kg} / \mathrm{m}^{2}\right)$ & $24.29 \pm 3.81$ & $24.39 \pm 3.88$ & 0.060 \\
\hline Paternal age (years) & $38.54 \pm 6.60$ & $38.41 \pm 6.26$ & 0.530 \\
\hline Total gonadotropin administered (IU) & $2477.61 \pm 986.76$ & $2453.75 \pm 770.55$ & 0.431 \\
\hline Estradiol level at hCG trigger day $(\mathrm{pg} / \mathrm{mL})$ & $1869.45 \pm 1797.94$ & $2060.45 \pm 1820.42$ & 0.051 \\
\hline Interval between hCG and oocyte retrieval (hours) & $35.34 \pm 0.41$ & $35.34 \pm 0.43$ & 0.733 \\
\hline \multicolumn{4}{|l|}{ cos outcomes* } \\
\hline Follicles $(n)$ & $14.80 \pm 0.22$ & $16.49 \pm 0.27$ & $<0.001$ \\
\hline Retrieved oocytes (n) & $10.09 \pm 0.16$ & $11.22 \pm 0.19$ & $<0.001$ \\
\hline Oocyte yield (\%) & $68.29 \pm 0.41$ & $69.71 \pm 0.52$ & 0.034 \\
\hline MII Oocytes (n) & $8.74 \pm 0.42$ & $6.97 \pm 0.52$ & $<0.001$ \\
\hline MI Oocytes (n) & $0.37 \pm 0.02$ & $2.28 \pm 0.25$ & $<0.001$ \\
\hline GV Oocytes (n) & $1.39 \pm 0.37$ & $1.27 \pm 0.45$ & 0.024 \\
\hline MII/oocytes (\%) & $86.23 \pm 0.37$ & $64.51 \pm 0.46$ & $<0.001$ \\
\hline MI/oocytes (\%) & $1.69 \pm 0.22$ & $24.87 \pm 0.27$ & $<0.001$ \\
\hline GV/oocytes (\%) & $11.82 \pm 0.32$ & $10.45 \pm 0.39$ & 0.007 \\
\hline \multicolumn{4}{|l|}{ Laboratorial outcomes** } \\
\hline Fertilization rate $(\%)$ & $83.15 \pm 0.44$ & $80.47 \pm 0.55$ & $<0.001$ \\
\hline Embryos obtained $(n)$ & $6.85 \pm 0.59$ & $6.35 \pm 0.074$ & $<0.001$ \\
\hline High-quality embryos rate at day $2(\%)$ & $27.0 \pm 0.50$ & $23.7 \pm 0.60$ & $<0.001$ \\
\hline High-quality embryos rate at day $3(\%)$ & $49.20 \pm 0.60$ & $47.70 \pm 0.70$ & 0.130 \\
\hline Blastocyst rate (\%) & $47.8 \pm 0.70$ & $47.5 \pm 0.80$ & 0.767 \\
\hline Transferred embryos ( $n$ ) & $1.69 \pm 0.23$ & $1.87 \pm 0.28$ & $<0.001$ \\
\hline Endometrial thickness ( $\mathrm{mm})$ & $10.62 \pm 0.53$ & $10.78 \pm 0.65$ & 0.046 \\
\hline \multicolumn{4}{|l|}{ Clinical outcomes*** } \\
\hline Implantation rate $(\%)$ & $32.24 \pm 2.37$ & $30.54 \pm 0.70$ & 0.038 \\
\hline Clinical pregnancy rate $(\%)$ & 43.72 & 22.25 & $<0.001$ \\
\hline Miscarriage rate $(\%)$ & 13.69 & 14.79 & 0.651 \\
\hline
\end{tabular}

hCG: human chorionic gonadotropin;

IU: international units.

* Data was adjusted for maternal age, maternal BMI and total FSH dose.

** Data was adjusted for maternal age, maternal BMI, total FSH dose, estradiol level at hCG trigger day and number of retrieved oocytes.

*** Data was adjusted for maternal age, maternal BMI, total FSH dose, estradiol level at hCG trigger day, number of retrieved oocytes, number of transferred embryos and endometrial thickness.

2006; Son et al., 2008; Wang et al., 2011), we noticed a small variance in the time interval comparing different cycles, so it was not possible to correlate this factor with immature oocyte incidence.

The rate of both MI and GV oocytes negatively impacted on the fertilization of the MII oocytes from the same cohort. In fact, increasing incidence of mature oocytes has been correlated with increasing fertilization rate (Halvaei et al., 2012; Kahraman et al., 2017). The oocyte competence depends on a multiplicity of factors; one of them is the ability to generate normal $\mathrm{Ca}^{2+}$ oscillation response induced by sperm penetration, that develops during the final stages of oocyte growth and maturation and is essential for promoting oocyte activation and fertilization initiation; thereafter MII oocytes that did not reach fully completed cytoplasmic maturity could have impairment in the acquisition of developmental competence (Cheung et al., 2000)

The fertilization of mature oocytes derived from cycles with heterogeneous follicular development can lead to abnormal early embryo development, with embryos with significantly more cleavages and lower blastocyst quality (Halvaei et al., 2012; Kahraman et al., 2017). Correlations of immature oocyte rates with lower cleave-stage quality and lower blastocyst formation were observed. However, the correlation of immature oocytes and embryo formation was not observed by others (Son et al., 2008).

The lower fertilization, high-quality embryo and blastocyst rates impacted directly on the implantation and 
pregnancy rates. We could establish a cut-off based on discriminant analysis of MI/oocyte rate of $10.5 \%$ for the prediction of pregnancy outcome. We observed that cycles with rate higher than $10.5 \%$ were correlated to higher response to ovarian stimulation, poor embryo development and almost two times lower pregnancy rate.

In conclusion the present study suggests that immature oocyte incidence is affected by the COS protocol and that immature oocyte incidence negatively impacts ICSI outcomes of mature oocytes from the same cohort. Our findings also demonstrated the importance of COS protocol and pituitary suppression type on the outcomes of ICSI cycles. And finally, cycles with lower than $10.5 \%$ of MI/ oocyte should be closed followed.

\section{CONFLICT OF INTEREST}

The authors have no conflict of interest to declare.

\section{Corresponding author:}

Edson Borges Jr.

Fertility Medical Group

São Paulo, SP - Brazil.

E-mail: edson@fertility.com.br

\section{REFERENCES}

Alpha Scientists in Reproductive Medicine and ESHRE Special Interest Group of Embryology. The Istanbul consensus workshop on embryo assessment: proceedings of an expert meeting. Hum Reprod. 2011;26:1270-83. PMID: 21502182 DOI: 10.1093/humrep/der037

Borges E Jr, Setti AS, Braga DP, Figueira RC, Iaconelli A Jr. Total motile sperm count has a superior predictive value over the WHO 2010 cut-off values for the outcomes of intracytoplasmic sperm injection cycles. Andrology. 2016;4:880-6. PMID: 27152971 DOI: 10.1111/andr.12199

Borges E Jr, Zanetti BF, Setti AS, Braga DP, Figueira RCS, Iaconelli A Jr. FSH dose to stimulate different patient' ages: when less is more. JBRA Assist Reprod. 2017;21:336-42. PMID: 29105483 DOI: 10.5935/1518-0557.20170058

Bosch E, Ezcurra D. Individualised controlled ovarian stimulation (iCOS): maximising success rates for assisted reproductive technology patients. Reprod Biol Endocrinol. 2011;9:82. PMID: 21693025 DOI: 10.1186/1477-78279-82

Braga DP, Figueira Rde C, Ferreira RC, Pasqualotto FF, Iaconelli A Jr, Borges $E$ Jr. Contribution of in-vitro maturation in ovarian stimulation cycles of poor-responder patients. Reprod Biomed Online. 2010;20:335-40. PMID: 20117048 DOI: 10.1016/j.rbmo.2009.12.009

Burgués S; Spanish Collaborative Group on Female Hypogonadotrophic Hypogonadism. The effectiveness and safety of recombinant human $\mathrm{LH}$ to support follicular development induced by recombinant human $\mathrm{FSH}$ in WHO group I anovulation: evidence from a multicentre study in Spain. Hum Reprod. 2001;16:2525-32. PMID: 11726569 DOI: $10.1093 /$ humrep/16.12.2525

Celik O, Celik N, Gungor S, Haberal ET, Aydin S. Selective Regulation of Oocyte Meiotic Events Enhances Progress in Fertility Preservation Methods. Biochem Insights. 2015;8:11-21. PMID: 26417205 DOI: 10.4137/BCI. S28596
Cheung A, Swann K, Carroll J. The ability to generate normal $\mathrm{Ca}(2+)$ transients in response to spermatozoa develops during the final stages of oocyte growth and maturation. Hum Reprod. 2000;15:1389-95. PMID: 10831575 DOI: $10.1093 /$ humrep/15.6.1389

Conti M, Hsieh M, Zamah AM, Oh JS. Novel signaling mechanisms in the ovary during oocyte maturation and ovulation. Mol Cell Endocrinol. 2012;356:65-73. PMID: 22101318 DOI: $10.1016 /$ j.mce.2011.11.002

Coticchio G, Dal Canto M, Mignini Renzini M, Guglielmo MC, Brambillasca F, Turchi D, Novara PV, Fadini R. Oocyte maturation: gamete-somatic cells interactions, meiotic resumption, cytoskeletal dynamics and cytoplasmic reorganization. Hum Reprod Update. 2015;21:427-54. PMID: 25744083 DOI: $10.1093 /$ humupd/dmv011

Devreker F, Pogonici E, De Maertelaer V, Revelard P, Van den Bergh M, Englert Y. Selection of good embryos for transfer depends on embryo cohort size: implications for the 'mild ovarian stimulation' debate. Hum Reprod. 1999;14:30028. PMID: 10601087 DOI: 10.1093/humrep/14.12.3002

Dor J, Seidman DS, Ben-Shlomo I, Levran D, Karasik A, Mashiach $S$. The prognostic importance of the number of oocytes retrieved and estradiol levels in poor and normal responders in in vitro fertilization (IVF) treatment. J Assist Reprod Genet. 1992;9:228-32. PMID: 1525451 DOI: $10.1007 / B F 01203818$

Downs SM. Nutrient pathways regulating the nuclear maturation of mammalian oocytes. Reprod Fertil Dev. 2015;27:572-82. PMID: 25798589 DOI: 10.1071/ RD14343

Emery BR, Wilcox AL, Aoki VW, Peterson CM, Carrell DT. In vitro oocyte maturation and subsequent delayed fertilization is associated with increased embryo aneuploidy. Fertil Steril. 2005;84:1027-9. PMID: 16213866 DOI: 10.1016/j. fertnstert.2005.04.036

Fatemi HM, Blockeel C, Devroey. Ovarian stimulation : today and tomorrow. Curr Pharm Biotechnol. 2012;13:3927. PMID: 21657998 DOI: $10.2174 / 138920112799362007$

Findlay JK, Hutt KJ, Hickey M, Anderson RA. How Is the Number of Primordial Follicles in the Ovarian Reserve Established? Biol Reprod. 2015;93:111. PMID: 26423124 DOI: $10.1095 /$ biolreprod.115.133652

Halvaei I, Ali Khalili M, Razi MH, Nottola SA. The effect of immature oocytes quantity on the rates of oocytes maturity and morphology, fertilization, and embryo development in ICSI cycles. J Assist Reprod Genet. 2012;29:803-10. PMID: 22644633 DOI: 10.1007/s10815-012-9799-6

Jaffe LA, Egbert JR. Regulation of Mammalian Oocyte Meiosis by Intercellular Communication Within the Ovarian Follicle. Annu Rev Physiol. 2017;79:237-60. PMID: 27860834 DOI: $10.1146 /$ annurev-physiol-022516-034102

Jungheim ES, Meyer M, Broughton DE. Best practices for controlled ovarian stimulation in IVF. Semin Reprod Med. 2015;33:77-82. PMID: 25734345 DOI: $10.1055 / \mathrm{s}-0035-$ 1546424 
Kahraman S, Cetinkaya CP, Cetinkaya M, Yelke H, Colakoglu YK, Aygun M, Montag M. The effect of follicle size and homogeneity of follicular development on the morphokinetics of human embryos. J Assist Reprod Genet. 2017;34:895903. PMID: 28470453 DOI: $10.1007 /$ s10815-017-0935-1

Mehlmann LM. Stops and starts in mammalian oocytes: recent advances in understanding the regulation of meiotic arrest and oocyte maturation. Reproduction. 2005;130:7919. PMID: 16322539 DOI: 10.1530/rep.1.00793

Messinis IE, Templeton AA. The importance of follicle-stimulating hormone increase for folliculogenesis. Hum Reprod. 1990;5:153-6. PMID: 2108988 DOI: 10.1093/oxfordjournals.humrep.a137060

Monniaux D, Clément F, Dalbiès-Tran R, Estienne A, Fabre $S$, Mansanet $C$, Monget $P$. the ovarian reserve of primordial follicles and the dynamic reserve of antral growing follicles: what is the link? Biol Reprod. 2014;90:85. PMID: 24599291 DOI: $10.1095 /$ biolreprod.113.117077

Nogueira D, Staessen C, Van de Velde H, Van Steirteghem A. Nuclear status and cytogenetics of embryos derived from in vitro-matured oocytes. Fertil Steril. 2000;74:295-8. PMID: 10927047 DOI: $10.1016 /$ S0015-0282(00)00642-7

Pacchiarotti A, Aragona C, Gaglione R, Selman H. Efficacy of a combined protocol of urinary and recombinant follicle-stimulating hormone used for ovarian stimulation of patients undergoing ICSI cycle. J Assist Reprod Genet. 2007;24:400-5. PMID: 17653846 DOI: 10.1007/s10815007-9159-0

Palermo G, Joris H, Devroey P, Van Steirteghem AC. Pregnancies after intracytoplasmic injection of single spermatozoon. Lancet. 1992;340:17-8. PMID: 1351601 DOI: 10.1016/0140-6736(92)92425-f

Papaleo E, Vanni VS, Viganò P, La Marca A, Pagliardini L, Vitrano R, Zanirato M, Marsiglio E, Privitera L, Candiani M. Recombinant LH administration in subsequent cycle after "unexpected" poor response to recombinant FSH monotherapy. Gynecol Endocrinol. 2014;30:813-6. PMID: 24968088 DOI: $10.3109 / 09513590.2014 .932342$

Raju GA, Chavan R, Deenadayal M, Gunasheela D, Gutgutia R, Haripriya G, Govindarajan M, Patel NH, Patki AS. Luteinizing hormone and follicle stimulating hormone synergy: A review of role in controlled ovarian hyper-stimulation. J Hum Reprod Sci. 2013;6:227-34. PMID: 24672160 DOI: $10.4103 / 0974-1208.126285$

Raziel A, Schachter M, Strassburger D, Kasterstein E, RonEl R, Friedler $S$. In vivo maturation of oocytes by extending the interval between human chorionic gonadotropin administration and oocyte retrieval. Fertil Steril. 2006;86:583-7. PMID: 16828475 DOI: 10.1016/j.fertnstert.2006.02.091
Santi D, Casarini L, Alviggi C, Simoni M. Efficacy of Follicle-Stimulating Hormone (FSH) Alone, FSH + Luteinizing Hormone, Human Menopausal Gonadotropin or FSH + Human Chorionic Gonadotropin on Assisted Reproductive Technology Outcomes in the "Personalized" Medicine Era: A Meta-analysis. Front Endocrinol (Lausanne). 2017;8:114. PMID: 28620352 DOI: 10.3389/fendo.2017.00114

Selman HA, De Santo M, Sterzik K, Coccia E, El-Danasouri I. Effect of highly purified urinary follicle-stimulating hormone on oocyte and embryo quality. Fertil Steril. 2002;78:1061-7. PMID: 12413994 DOI: 10.1016/S00150282(02)04202-4

Selman H, Pacchiarotti A, El-Danasouri I. Ovarian stimulation protocols based on follicle-stimulating hormone glycosylation pattern: impact on oocyte quality and clinical outcome. Fertil Steril. 2010;94:1782-6. PMID: 19939369 DOI: $10.1016 /$ j.fertnstert.2009.10.005

Shu Y, Gebhardt J, Watt J, Lyon J, Dasig D, Behr B. Fertilization, embryo development, and clinical outcome of immature oocytes from stimulated intracytoplasmic sperm injection cycles. Fertil Steril. 2007;87:1022-7. PMID: 17261289 DOI: $10.1016 /$ j.fertnstert.2006.08.110

Son WY, Chung JT, Chian RC, Herrero B, Demirtas E, Elizur S, Gidoni Y, Sylvestre C, Dean N, Tan SL. A 38 h interval between hCG priming and oocyte retrieval increases in vivo and in vitro oocyte maturation rate in programmed IVM cycles. Hum Reprod. 2008;23:2010-6. PMID: 18556681 DOI: $10.1093 /$ humrep/den 210

Suchanek E, Huderer K, Dobec D, Hlavati V, Simunic V, Grizelj V. Number of follicles, oocytes and embryos in human in vitro fertilization is relative to serum estradiol and progesterone patterns during different types of ovarian hyperstimulation. Eur J Obstet Gynecol Reprod Biol. 1994;56:121-7. PMID: 7805963 DOI: 10.1016/00282243(94)90268-2

Thomas FH, Ethier JF, Shimasaki S, Vanderhyden BC. Follicle-Stimulating Hormone Regulates Oocyte Growth by Modulation of Expression of Oocyte and Granulosa Cell Factors. Endocrinology. 2005;146:941-9. PMID: 15539559 DOI: $10.1210 /$ en.2004-0826

van der Meer M, Hompes PG, Scheele F, Schoute E, Veersema S, Schoemaker J. Follicle stimulating hormone (FSH) dynamics of low dose step-up ovulation induction with FSH in patients with polycystic ovary syndrome. Hum Reprod. 1994;9:1612-7. PMID: 7836509 DOI: 10.1093/oxfordjournals.humrep.a138761

van Santbrink EJ, Hop WC, van Dessel TJ, de Jong FH, Fauser BC. Decremental follicle-stimulating hormone and dominant follicle development during the normal menstrual cycle. Fertil Steril. 1995;64:37-43. PMID: 7789578 DOI: $10.1016 /$ S0015-0282(16)57652-3

Wang W, Zhang $\mathrm{XH}$, Wang WH, Liu YL, Zhao $\mathrm{LH}$, Xue SL, Yang $\mathrm{KH}$. The time interval between hCG priming and oocyte retrieval in ART program: a meta-analysis. J Assist Reprod Genet. 2011;28:901-10. PMID: 21792666 DOI: $10.1007 / \mathrm{s} 10815-011-9613-\mathrm{x}$ 